\title{
Tendências de acesso e utilização dos serviços de saúde na APS entre idosos no Brasil nos anos 2008, 2013 e 2019
}

\author{
Trends in the access and use of health services in PHC \\ among Brazilian older adults in the years 2008, 2013 and 2019
}

\begin{abstract}
Vanovya Alves Claudino Cesário (https://orcid.org/0000-0002-9707-8554) ${ }^{1}$
Marquiony Marques dos Santos (https://orcid.org/0000-0001-5812-4004) ${ }^{2}$

Tamires Carneiro de Oliveira Mendes (https://orcid.org/0000-0002-7254-4096) ${ }^{3}$

Paulo Roberto Borges de Souza Júnior (https://orcid.org/0000-0002-8142-4790) ${ }^{4}$

Kenio Costa de Lima (https://orcid.org/0000-0002-5668-4398) ${ }^{5}$
\end{abstract}

${ }^{1}$ Programa de Pós-

Graduação em Ciências da Saúde, Universidade Federal do Rio Grande do Norte. Av. Sen. Salgado Filho 3000 , Lagoa Nova. 59064-720 Natal RN Brasil.

vanovya@gmail.com.

${ }^{2}$ Universidade do Estado do Rio Grande do Norte. Caicó RN Brasil.

${ }^{3}$ Conselho Estadual dos Direitos da Pessoa Idosa do Rio Grande do Norte. Natal RN Brasil.

${ }^{4}$ Instituto de Comunicação e Informação Científica e Tecnológica em Saúde, Fundação Oswaldo Cruz. Rio de Janeiro RJ Brasil.

${ }^{5}$ Programa de Pós-

Graduação em Saúde

Coletiva, Universidade

Federal do Rio Grande do

Norte. Natal RN Brasil.

\begin{abstract}
This paper aims to identify the conditions and trends in access and use of primary health care (PHC) services by Brazilian older adults in 2008, 2013, and 2019. We performed a cross-sectional panel study with a descriptive analysis of the percentages and confidence intervals of the variables listed, in which data on the elderly population investigated in the Health Supplement of the 2008 National Household Sample Survey (PNAD) and the 2013 and 2019 National Health Surveys (PNS) were obtained. We initially identified that, while PHC was the primary service sought for care needs, this demand tended to decline in all Brazilian regions, among older women, the 60-69 years age group, and whites. The number of households registered with the USF increased 15.2\%; the search for a place, service, or health professional by 4.5\%; and the population served by $31.4 \%$. The main factors of non-attendance were not being able to get a vacancy/service ticket and doctor unavailability. A growing trend was observed among those diagnosed with Systemic Arterial Hypertension, Diabetes Mellitus, and multimorbidities among those investigated.
\end{abstract}

Key words Aged, Health services accessibility, Primary health care
Resumo $O$ artigo tem por objetivo contribuir na identificação das condições e tendências de acesso e utilização dos serviços da atenção primária em saúde (APS) pelos idosos brasileiros nos anos de 2008, 2013 e 2019. Um estudo transversal em painéis com análise descritiva dos percentuais e intervalos de confiança das variáveis elencadas, no qual foram resgatados os dados relativos à população idosa investigada no Suplemento Saúde da Pesquisa Nacional por Amostra de Domicílios (PNAD) do ano 2008 e da Pesquisa Nacional de Saúde (PNS) 2013 e 2019. Inicialmente identificou-se que, apesar da APS ter sido o principal serviço procurado mediante a necessidade de assistência, houve uma tendência de redução para esta procura em todas as regiões brasileiras, principalmente entre as mulheres idosas, na faixa etária de 60-69 anose na população de raça/cor branca. Verificou-se um aumento de 15,2\% no quantitativo de domicílios cadastrados na Unidade de Saúde da Família; 4,5\% na procura por lugar, serviço ou profissional de saúde; e de 31,4\% na população atendida. Os principais fatores de não atendimento foram não conseguir vaga/senha e não ter médico atendendo. $E$, dentre os investigados, verificou-se uma tendência de crescimento entre os que tinham o diagnóstico de hipertensão arterial sistêmica, diabetes mellitus e multimorbidades.

Palavras-chave Idoso, Acesso aos serviços de saúde, Atenção primária à saúde 


\section{Introdução}

O crescimento das Doenças Crônicas Não Transmissíveis (DCNT) no perfil epidemiológico da população brasileira e mundial constitui um dos maiores problemas globais de saúde, especialmente entre as pessoas idosas. Doenças como hipertensão arterial sistêmica, diabetes mellitus e problemas osteoarticulares que, em mais da metade dos indivíduos idosos, se apresentam em situação de multimorbidades (ocorrência simultânea de $\geq 2$ ou $\geq 3$ doenças crônicas), provocam limitações e incapacidade para atividades laborais e da vida diária, afetam a qualidade de vida por um longo período e podem levar a mortalidade prematura ${ }^{1-4}$.

$\mathrm{Na}$ atenção à pessoa idosa, um modelo assistencial fragmentado com multiplicação de consultas especializadas e sem coordenação do cuidado, informação não compartilhada, aumento da polifarmácia, repetições de exames e outros procedimentos sobrecarrega o sistema de saúde, provoca iatrogenias e não atende adequadamente às necessidades em saúde dos usuários. Assim, $\mathrm{o}$ atual perfil epidemiológico brasileiro demanda um modelo que priorize as instâncias leves de cuidado, a Atenção Primária à Saúde (APS), com ações de promoção, considerando a educação e participação ativa dos sujeitos, a prevenção e retardamento de doenças e fragilidades, bem como a manutenção da independência e da autonomia, contribuindo para que a maior longevidade conquistada pelas gerações atuais possa ser desfrutada com qualidade de vida ${ }^{5,6}$.

A necessidade de fortalecimento e priorização da APS resgata sua relevância no SUS, ao ser definida como porta de entrada preferencial e centro de comunicação com a rede de atenção em saúde, e reforça sua importância na garantia da igualdade no acesso e estímulo à utilização dos serviços de saúde ${ }^{7}$.

$\mathrm{O}$ acesso constitui a entrada do usuário no serviço de saúde mediante necessidade constatada pelo indivíduo. Esse deve ter caráter universal e promover impactos substanciais na atenção em saúde, tais como a redução de iniquidades, fortalecimento do SUS, identificação e redirecionamento de ações, mediante as particularidades dos grupos populacionais, como os idosos; além de favorecer a adequada utilização dos serviços, a qual consiste no contato direto ou indireto com os serviços de saúde, através de consultas, hospitalizações, ações preventivas e exames diagnósticos ${ }^{8-11}$.

A realidade vigente demonstra que os fatores sociodemográficos têm íntima relação com o acesso e utilização dos serviços em saúde e, portanto, exige uma análise fidedigna da atenção à pessoa idosa, como as possíveis diferenças de gênero, raça, faixa etária e região de residência ${ }^{12,13}$. Nesse contexto, os dados provenientes do Suplemento Saúde da Pesquisa Nacional por Amostra de Domicílios (PNAD) e a Pesquisa Nacional de Saúde (PNS) permitem uma análise aprofundada da casuística, considerando as mais diversas perspectivas, desde usuários que não tentaram a entrada no serviço até os que foram adequadamente atendidos.

Dessa forma, espera-se que o presente estudo possa contribuir na identificação das condições e tendências de acesso e utilização dos serviços de saúde da APS no Brasil pelos idosos_nos anos de 2008, 2013 e 2019, ao identificar as similaridades e disparidades conforme as variáveis estudadas nos períodos em questão.

\section{Metodologia}

Trata-se de um estudo transversal em painéis, no qual realizou-se uma análise da série histórica dos indicadores de acesso e utilização dos serviços de saúde da APS a partir de dados do Suplemento Saúde da Pesquisa Nacional por Amostra de Domicílios (PNAD) do ano 2008 e da Pesquisa Nacional de Saúde (PNS) dos anos 2013 e 2019. Estes inquéritos domiciliares nacionais foram conduzidos pelo Instituto Brasileiro de Geografia e Estatística (IBGE), em parceria com o Ministério da Saúde, sendo a PNS uma continuação dos suplementos da PNAD, com maior detalhamento e especificidade da metodologia para o tema da saúde ${ }^{14}$.

A PNAD e a PNS são estudos de base populacional, representativos para o país, grandes regiões, Unidades da Federação, capitais e regiões metropolitanas ${ }^{15}$. Para o presente estudo foram considerados os dados relativos à população idosa, a partir de 60 anos.

Os indicadores foram calculados a partir das perguntas em comum nos diferentes inquéritos (Quadro 1), as quais foram utilizadas nos questionários de modo a garantir a manutenção da comparabilidade dos dados na série histórica. Para analisar o acesso e utilização dos serviços de saúde na APS foram consideradas as variáveis que se referiam ao seguimento do tratamento pelo serviço/profissional, serviço de saúde que costuma procurar quando precisa, procura recente por serviço de saúde, tipo de serviço de saúde procurado, dificuldade de acesso ao servi- 
Quadro 1. Perguntas apresentadas nos diferentes inquéritos.

\begin{tabular}{|c|c|c|c|}
\hline Variável do estudo & PNAD 2008 & PNS 2013 & PNS 2019 \\
\hline $\begin{array}{l}\text { Cadastro na } \\
\text { Unidade de Saúde } \\
\text { da Família }\end{array}$ & $\begin{array}{l}\text { V0233 - O seu domicílio } \\
\text { está cadastrado } \\
\text { (registrado) na unidade de } \\
\text { Saúde da Família? }\end{array}$ & $\begin{array}{l}\text { B1 - O seu domicílio está } \\
\text { cadastrado na unidade de } \\
\text { saúde da família? }\end{array}$ & $\begin{array}{l}\text { B1 - O seu domicílio está } \\
\text { cadastrado na unidade de } \\
\text { saúde da família? }\end{array}$ \\
\hline $\begin{array}{l}\text { Prevalência de } \\
\text { Diabetes }\end{array}$ & $\begin{array}{l}\text { V1312 - Algum médico ou } \\
\text { profissional de saúde } \\
\text { já disse que__ tem } \\
\text { diabetes? }\end{array}$ & $\begin{array}{l}\text { Q30 - Algum médico já } \\
\text { lhe deu o diagnóstico de } \\
\text { diabetes? }\end{array}$ & $\begin{array}{l}\text { Q30a - Algum médico já lhe } \\
\text { deu o diagnóstico de diabetes? }\end{array}$ \\
\hline $\begin{array}{l}\text { Prevalência de } \\
\text { Hipertensão } \\
\text { arterial }\end{array}$ & $\begin{array}{l}\text { V1314 - Algum médico } \\
\text { ou profissional de saúde } \\
\text { já disse que __ tem } \\
\text { hipertensão (pressão alta)? }\end{array}$ & $\begin{array}{l}\text { Q2 - Algum } \\
\text { médico já lhe deu } \\
\text { o diagnóstico de } \\
\text { hipertensão arterial } \\
\text { (pressão alta)? }\end{array}$ & $\begin{array}{l}\text { Q2a - Algum médico já } \\
\text { lhe deu o diagnóstico de } \\
\text { hipertensão arterial (pressão } \\
\text { alta)? }\end{array}$ \\
\hline $\begin{array}{l}\text { Seguimento } \\
\text { do tratamento } \\
\text { pelo serviço/ } \\
\text { profissional }\end{array}$ & $\begin{array}{l}\text { V1345 - _ costuma } \\
\text { procurar o mesmo lugar, } \\
\text { mesmo médico ou mesmo } \\
\text { serviço de } \\
\text { saúde quando precisa de } \\
\text { atendimento de saúde? }\end{array}$ & $\begin{array}{l}\mathrm{J} 9 \text { - __ costuma } \\
\text { procurar o mesmo } \\
\text { lugar, mesmo médico } \\
\text { ou mesmo serviço de } \\
\text { saúde quando precisa de } \\
\text { atendimento de saúde? }\end{array}$ & $\begin{array}{l}\text { J9 - __ costuma procurar } \\
\text { o mesmo lugar, mesmo } \\
\text { médico ou mesmo serviço } \\
\text { de saúde quando precisa de } \\
\text { atendimento de saúde? }\end{array}$ \\
\hline $\begin{array}{l}\text { Serviço de saúde } \\
\text { que costuma } \\
\text { procurar quando } \\
\text { precisa }\end{array}$ & $\begin{array}{l}\text { V1346 - Quando está } \\
\text { doente ou precisando de } \\
\text { atendimento de saúde } \\
\text { costuma procurar: }\end{array}$ & $\begin{array}{l}\text { J10 - Quando está } \\
\text { doente ou precisando de } \\
\text { atendimento de saúde } \\
\text { costuma procurar: }\end{array}$ & $\begin{array}{l}\text { J10a - Quando está doente ou } \\
\text { precisando de atendimento } \\
\text { de saúde___ costuma } \\
\text { procurar: }\end{array}$ \\
\hline Variável do estudo & PNAD 2008 & PNS 2013 & PNS 2019 \\
\hline $\begin{array}{l}\text { Procura recente } \\
\text { por serviço de } \\
\text { saúde }\end{array}$ & $\begin{array}{l}\text { V1350 - Nas duas últimas } \\
\text { semanas,__ procurou } \\
\text { algum lugar, serviço ou } \\
\text { profissional de saúde para } \\
\text { atendimento relacionado à } \\
\text { própria saúde? }\end{array}$ & $\begin{array}{l}\text { J14 - Nas duas últimas } \\
\text { semanas, __ procurou } \\
\text { algum lugar, serviço ou } \\
\text { profissional de saúde para } \\
\text { atendimento relacionado } \\
\text { à própria saúde? }\end{array}$ & $\begin{array}{l}\text { J14 - Nas duas últimas } \\
\text { semanas, _ procurou algum } \\
\text { lugar, serviço ou profissional } \\
\text { de saúde para atendimento } \\
\text { relacionado à própria saúde? }\end{array}$ \\
\hline $\begin{array}{l}\text { Tipo de serviço de } \\
\text { saúde }\end{array}$ & $\begin{array}{l}\text { V1357 - Onde } \\
\text { procurou o último } \\
\text { atendimento de saúde por } \\
\text { este mesmo motivo nas } \\
\text { duas últimas semanas? }\end{array}$ & $\begin{array}{l}\text { J20 - Onde _ procurou } \\
\text { o último atendimento de } \\
\text { saúde por este motivo nas } \\
\text { duas últimas semanas? }\end{array}$ & $\begin{array}{l}\text { J20a - Onde _ procurou o } \\
\text { último atendimento de saúde } \\
\text { por este motivo nas duas } \\
\text { últimas semanas? }\end{array}$ \\
\hline $\begin{array}{l}\text { Dificuldade de } \\
\text { acesso ao serviço } \\
\text { de saúde }\end{array}$ & $\begin{array}{l}\text { V1358- Nessa última } \\
\text { vez que procurou } \\
\text { atendimento de } \\
\text { saúde, nas duas últimas } \\
\text { semanas, } \overline{\text { atendido(a)? foi }} \\
\end{array}$ & $\begin{array}{l}\text { J21 - Nessa última vez que } \\
\text { procurou atendimento de } \\
\text { saúde, nas duas últimas } \\
\text { semanas, foi } \\
\text { atendido(a)? }\end{array}$ & $\begin{array}{l}\text { J21 - Nessa última vez que } \\
\text { procurou atendimento de } \\
\text { saúde por este motivo, nas } \\
\text { duas últimas semanas, __ foi } \\
\text { atendido(a)? }\end{array}$ \\
\hline $\begin{array}{l}\text { Motivo por não } \\
\text { atendimento em } \\
\text { última consulta }\end{array}$ & $\begin{array}{l}\text { V1359a - Por que } \\
\text { motivo não } \\
\text { foi atendido(a) nessa } \\
\text { última vez que procurou } \\
\text { atendimento de saúde nas } \\
\text { duas últimas semanas? }\end{array}$ & $\begin{array}{l}\text { J22 - Por que motivo } \\
\text { não foi } \\
\text { atendido(a) nessa } \\
\text { última vez que procurou } \\
\text { atendimento de saúde nas } \\
\text { duas últimas semanas? }\end{array}$ & $\begin{array}{l}\text { J22a - Por que motivo } \\
\text { não foi atendido(a) nessa } \\
\text { última vez que procurou } \\
\text { atendimento de saúde nas } \\
\text { duas últimas semanas? }\end{array}$ \\
\hline
\end{tabular}

Fonte: Pesquisa Nacional por Amostra de Domicílios (2008). Pesquisa Nacional de Saúde 2013 e 2019.

ço de saúde e motivo por não atendimento em última consulta, explicitadas no Quadro 1.
Os indicadores de morbidade foram baseados nas variáveis prevalência de diabetes mellitus; Pre- 
valência de hipertensão arterial sistêmica e prevalência de multimorbidades (com a presença de 2 ou mais das seguintes patologias: doenças crônicas de coluna, artrite reumatóide, câncer, diabetes mellitus, asma/bronquite, hipertensão arterial sistêmica, doenças do coração, insuficiência renal crônica, colesterol alto). Enquanto a cobertura da APS baseou-se na variável cadastro na Unidade de Saúde da Família, conforme Quadro 1.

As pessoas idosas investigadas nos três inquéritos foram caracterizadas quanto ao sexo, faixa etária e cor/raça. A série histórica dos indicadores de perfil/necessidade de saúde e de acesso e utilização foi analisada por frequências e intervalos de confiança no nível nacional e das grandes regiões a partir das estimativas das amostras complexas. Posteriormente, foi realizado o teste de Wald com correção de Rao-Scott para encontrar diferenças entre as proporções das variáveis categóricas nas PNAD 2008, PNS 2013 e PNS 2019, por considerar o peso amostral e o efeito do desenho nos cálculos.

\section{Resultados}

O número de idosos entrevistados na PNAD 2008 foi de 26.350, enquanto da PNS 2013 e 2019 foram de 23.815 e 43.554 , respectivamente. A Figura 1 apresenta a distribuição dos idosos que costumam procurar pela Atenção Primária em Saúde, a qual, apesar de permanecer como o principal serviço procurado, teve uma diminuição de 53,2\% em 2008 para 46,2\% em 2019. Por outro lado, os resultados apresentam um aumento da busca pelos serviços de pronto atendimento público ou privado, variando de 4,6\% na PNAD 2008, para $14,4 \%$ na PNS 2013 e 14,6\% na PNS 2019, enquanto os demais tipos de serviços de saúde tiveram números similares ao longo do período.

A Tabela 1 avalia o percentual de idosos que procuram a APS, estratificada por sexo, faixa etária, raça e região Nessa avaliação há uma diminuição em ambos os sexos, porém, no sexo feminino há uma redução de quase 8 pontos percentuais da PNAD 2008 para a PNS 2019. A queda da procura pela APS também é observada em relação à faixa etária, sendo mais evidente na faixa etária de 60 a 69 anos, que passa de 55,7\% para $47,80 \%$ no período de 2008 para 2019, respectivamente.

A tendência da procura pela APS varia entre as categorias de cor/raça no período avaliado. Os idosos de cor/raça preta passaram a buscar menos a APS no período da PNAD 2008 para a PNS
2013, mas houve um aumento para a PNS 2019. Outro destaque é a maior queda entre os idosos que se autodeclararam de raça/cor branca, passando de $46,1 \%$ para $38,8 \%$, conforme apresentado na Tabela 1. A procura do idoso pela APS também varia em relação à região de moradia, com uma redução em todas as regiões no período de 2008 a 2013, tendência esta que permaneceu em 2019 no Sudeste, enquanto nas demais regiões houve um discreto aumento (Tabela 1).

Apesar da redução na procura pelos serviços de APS, verificou-se que o percentual de domicílios cadastrados na Unidade de Saúde da Família (USF) aumentou consideravelmente, aproximadamente 12 pontos percentuais, da PNAD 2008 para a PNS 2013. Ao passo que entre 2008 e 2019 houve um aumento de $4,5 \%$ na procura recente por lugar, serviço ou profissional de saúde; e de aproximadamente 31 pontos percentuais entre os usuários atendidos (Tabela 2).

Os principais motivos de não atendimento pelo serviço de APS permaneceram praticamente os mesmos, não conseguir vaga/senha e não ter médico atendendo, de forma que esta última casuística apresentou uma tendência de redução de 18 pontos percentuais entre 2008 e 2019, conforme Tabela 2 As regiões brasileiras compartilham desta tendência, com destaque para os idosos do Nordeste em 2013 (34\%) e Centro-Oeste em 2019 (58,9\%) terem referido como motivação do não atendimento o fato de que esperou muito $e$ desistiu de conseguir o acesso e utilização do serviço. Nas regiões Norte em 2008 e 2019 e Sudeste, em 2019, há, respectivamente, um relevante quantitativo de $34,3 \% ; 25,7 \%$; e $20,6 \%$ de idosos que referiram não ter serviço ou profissional especializado como fator determinante para a não realização do seu atendimento (Tabela 3 ).

No tocante às morbidades verifica-se uma tendência de aumento nos casos de idosos atendidos pela APS que tem o diagnóstico de hipertensão arterial, diabetes mellitus e multimorbidades, com destaque para o crescimento de aproximadamente 12 pontos percentuais neste último, conforme verificado na Tabela 2. No âmbito regional, verifica-se na Tabela 3 diferenças significativas apenas nas regiões Nordeste e Centro-oeste para o diagnóstico de hipertensão, com um aumento de cinco e sete pontos percentuais, respectivamente. $\mathrm{O}$ número de idosos com Diabetes e multimorbidades apresentou uma tendência de aumento em todas as regiões brasileiras, com destaque para as multimorbidades na região Sul e Sudeste que apresentaram um crescimento de $10,2 \%$ e de $13,1 \%$, respectivamente. 


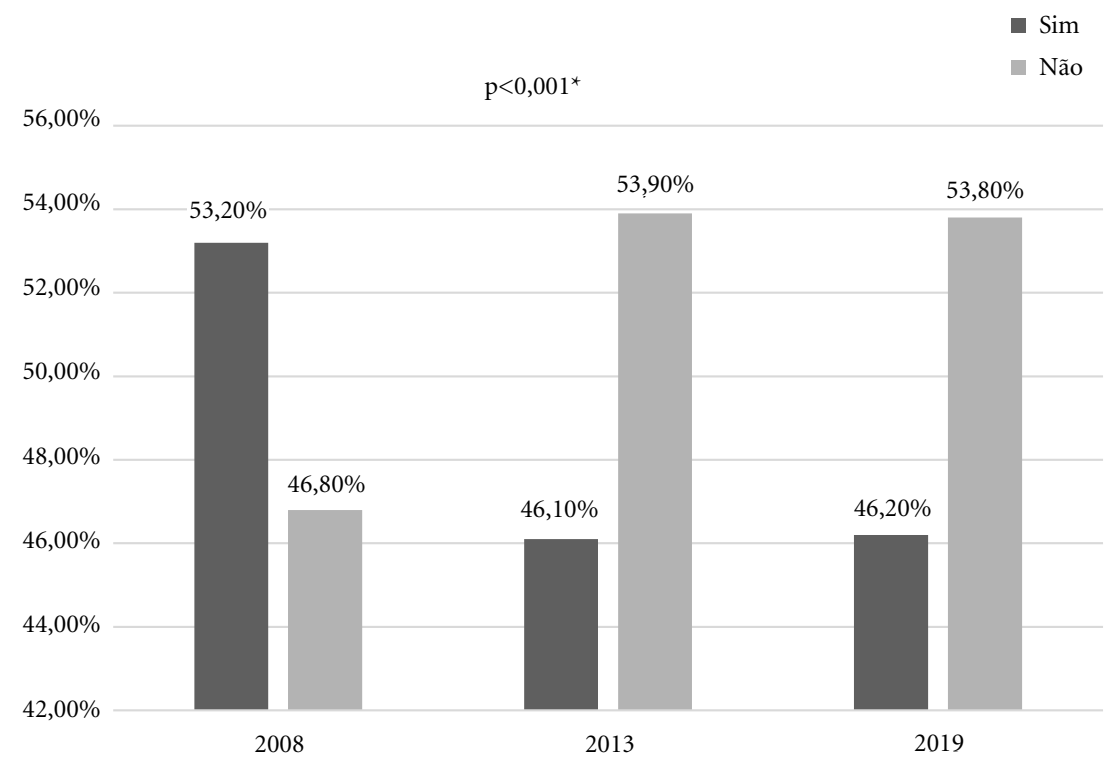

Figura 1. Distribuição dos idosos que costumam procurar pela Atenção Primária em Saúde no Brasil, 2019.

Fonte: Pesquisa Nacional por Amostra de Domicílios (2008). Pesquisa Nacional de Saúde 2013 e 2019.

Tabela 1. Percentual de idosos que procuram a APS, estratificado por sexo, faixa etária, cor/raça e região no Brasil, 2019.

\begin{tabular}{|c|c|c|c|c|}
\hline & $\begin{array}{c}2008 \\
(n=26350 \\
\%(I C 95 \%)\end{array}$ & $\begin{array}{c}2013 \\
(\mathrm{n}=23815) \\
\%(\mathrm{IC} 95 \%)\end{array}$ & $\begin{array}{c}2019 \\
(\mathrm{n}=43554) \\
(\mathrm{IC} 95 \%)\end{array}$ & Valor de $p$ \\
\hline \multicolumn{5}{|l|}{ Sexo } \\
\hline Homem & $53,5(52,0-54,8)$ & $46,6(44,7-48,6)$ & $47,3(45,2-48,3)$ & $<0,001$ \\
\hline Mulher & $53,0(52,5-55,4)$ & $45,6(43,6-47,4)$ & $45,3(43,2-46,0)$ & $<0,001$ \\
\hline \multicolumn{5}{|l|}{ Faixa etária } \\
\hline 60 a 69 anos & $55,7(54,4-57,0)$ & $47,6(45,6-49,6)$ & $47,8(45,7-48,7)$ & $<0,001$ \\
\hline 70 a 79 anos & $52,0(51,2-54,3)$ & $46,1(43,7-48,5)$ & $45,8(43,4-47,0)$ & $<0,001$ \\
\hline 80 anos ou mais & $45,8(44,2-49,1)$ & $39,8(36,6-43,3)$ & $40,3(37,4-42,5)$ & $<0,001$ \\
\hline \multicolumn{5}{|l|}{ Cor/Raça } \\
\hline Branca & $46,1(44,7-47,5)$ & $40,8(38,5-43,0)$ & $38,8(36,6-39,9)$ & $<0,001$ \\
\hline Preta & $66,5(63,7-69,2)$ & $48,5(43,5-52,9)$ & $55,3(51,9-58,0)$ & $<0,001$ \\
\hline Amarela & $27,8(23,6-39,1)$ & $22,5(14,0-34,0)$ & $38,9(28,2-45,7)$ & 0,054 \\
\hline Parda & $63,5(62,0-65,3)$ & $54,5(52,2-56,7)$ & $54,4(52,1-55,3)$ & $<0,001$ \\
\hline Indígena & $58,7(40,6-64,9)$ & $43,2(25,7-63,5)$ & $51,4(38,7-62,0)$ & 0,388 \\
\hline \multicolumn{5}{|l|}{ Região } \\
\hline Norte & $54,8(51,0-61,3)$ & $52,0(47,7-56,1)$ & $51,2(48,2-53,5)$ & 0,502 \\
\hline Nordeste & $59,4(56,5-61,8)$ & $51,1(48,0-53,8)$ & $52,3(49,8-53,6)$ & $<0,001$ \\
\hline Sudeste & $48,5(47,8-51,0)$ & $41,3(38,6-44,2)$ & $39,1(36,3-40,7)$ & $<0,001$ \\
\hline Sul & $57,7(54,8-60,4)$ & $52,1(48,2-56,0)$ & $55,3(52,0-56,8)$ & 0,069 \\
\hline Centro-oeste & $53,3(49,7-56,3)$ & $44,6(41,0-48,1)$ & $48,0(43,9-50,9)$ & 0,001 \\
\hline
\end{tabular}

Fonte: Pesquisa Nacional por Amostra de Domicílios (2008). Pesquisa Nacional de Saúde 2013 e 2019. 
Tabela 2. Descrição temporal das variáveis relativas a morbidades, acesso e utilização de serviços de saúde na população idosa brasileira, 2019.

\begin{tabular}{|c|c|c|c|c|}
\hline & $\begin{array}{c}2008 \\
(\mathrm{n}=26350) \\
\%(\mathrm{IC} 95 \%)\end{array}$ & $\begin{array}{c}2013 \\
(\mathbf{n}=23815) \\
\%(I C 95 \%)\end{array}$ & $\begin{array}{c}2019 \\
(\mathrm{n}=43554) \\
\%(\mathrm{IC} 95 \%)\end{array}$ & $\begin{array}{l}\text { Valor } \\
\text { de p }\end{array}$ \\
\hline \multicolumn{5}{|c|}{ O seu domicílio está cadastrado (registrado) na unidade de Saúde da Família? } \\
\hline Sim & $61,7(60,1-63,3)$ & $73,3(71,2-75,3)$ & $76,9(75-78,6)$ & $<0,001$ \\
\hline Não & $38,3(36,7-39,9)$ & $19,7(18-21,5)$ & $16,3(14,7-18)$ & \\
\hline Não sabe & - & $7,0(6-8,2)$ & $6,9(6,1-7,8)$ & \\
\hline \multicolumn{5}{|c|}{$\begin{array}{l}\text { Nas duas últimas semanas, __ procurou algum lugar, serviço ou profissional de saúde para } \\
\text { atendimento relacionado à própria saúde? }\end{array}$} \\
\hline Sim & $22,3(21,4-23,2)$ & $25,6(24,1-27,3)$ & $26,8(25,6-28)$ & $<0,001$ \\
\hline Não & $77,7(76,8-78,6)$ & $74,4(72,7-75,9)$ & $73,2(72-74,4)$ & \\
\hline \multicolumn{5}{|c|}{$\begin{array}{l}\text { Nessa última vez que procurou atendimento de saúde, nas duas últimas semanas, } \ldots \ldots \text { foi } \\
\text { atendido(a)? }\end{array}$} \\
\hline Sim & $55,4(41,5-68,4)$ & $57,9(38,7-75,0)$ & $86,8(82,8-90,0)$ & $<0,001$ \\
\hline Não & $44,6(31,6-58,5)$ & $42,1(25,0-61,3)$ & $13,2(10,0-17,2)$ & \\
\hline \multicolumn{5}{|c|}{$\begin{array}{l}\text { Por que motivo __ não foi atendido(a) nessa última vez que procurou atendimento de saúde } \\
\text { nas duas últimas semanas? }\end{array}$} \\
\hline Não conseguiu vaga ou senha & $21,5(9,6-41,2)$ & $24,7(11,1-46,1)$ & $26,8(17,8-38,2)$ & 0,015 \\
\hline Não tinha médico atendendo & $57,7(38,5-74,9)$ & $64,9(42,5-82,2)$ & $39,5(27,5-52,9)$ & \\
\hline $\begin{array}{l}\text { Não tinha serviço ou profissional } \\
\text { especializado }\end{array}$ & $12,8(4,5-31,3)$ & - & $9,7(4,1-21,4)$ & \\
\hline $\begin{array}{l}\text { O serviço ou equipamento não } \\
\text { estava funcionando }\end{array}$ & $3,1(0,4-19,1)$ & $0,5(0,1-3,4)$ & $2,3(0,7-6,8)$ & \\
\hline $\begin{array}{l}\text { Os equipamentos do serviço de } \\
\text { saúde não estavam funcionando } \\
\text { ou disponíveis para uso }\end{array}$ & - & $1,4(0,2-9,9)$ & $6,2(1,9-18,6)$ & \\
\hline Esperou muito e desistiu & $3,4(0,5-20,7)$ & $5,3(1,4-18,1)$ & $9,6(4,5-19,2)$ & \\
\hline Outro motivo & $1,5(0,2-9,9)$ & $3,3(0,5-18,6)$ & $6,0(2,4-14,4)$ & \\
\hline \multicolumn{5}{|l|}{ Diabetes } \\
\hline Não & $83(82,2-83,8)$ & $79(76,7-81,1)$ & $77,4(75,8-78,9)$ & $<0,001$ \\
\hline Sim & $17(16,2-17,8)$ & $21(18,9-23,3)$ & $22,6(21,1-24,2)$ & \\
\hline \multicolumn{5}{|l|}{ Hipertensão } \\
\hline Não & $42,9(41,7-44,0)$ & $44,6(41,8-47,5)$ & $41,1(39,3-43,0)$ & $<0,001$ \\
\hline Sim & $57,1(56,0-58,3)$ & $55,4(52,5-58,2)$ & $58,9(57,0-60,7)$ & \\
\hline \multicolumn{5}{|l|}{ Multimorbidade } \\
\hline Não & $50,0(48,9-51,2)$ & $42,9(40,0-45,9)$ & $38,5(36,7-40,2)$ & $<0,001$ \\
\hline Sim & $50,0(48,8-51,1)$ & $57,1(54,1-60,0)$ & $61,5(59,8-63,3)$ & \\
\hline
\end{tabular}

Fonte: Pesquisa Nacional por Amostra de Domicílios (2008). Pesquisa Nacional de Saúde 2013 e 2019.

\section{Discussão}

Os resultados encontrados demonstram que ao longo dos anos analisados verificou-se o aumento no acesso e utilização dos serviços de saúde da APS pelos idosos do Brasil, o que pôde ser observado em todas as regiões brasileiras, em ambos os sexos, em todas as faixas etárias, com destaque para as mulheres, os idosos na faixa etária de 60 a 69 anos, os que apresentam morbidades e os residentes na região Centro-Oeste.
Dentre tais resultados foram verificadas maiores disparidades entre a PNAD e as duas edições da PNS, o que pode estar relacionado a questões metodológicas, como o favorecimento de um efeito de plano de amostragem maior na PNAD devido ao maior quantitativo de domicílios incluídos na amostra. Destaca-se, ainda, que a PNS permitiu a desvinculação das informações de saúde da PNAD Contínua e sua inclusão no SIPD, além de possuir um desenho específico e exclusivamente direcionado para coleta de dados de saú- 
Tabela 3. Descrição temporal das variáveis relativas às morbidades, acesso e utilização de serviços de saúde na população idosa brasileira, por região geográfica, 2019.

\begin{tabular}{|c|c|c|c|c|}
\hline & $\begin{array}{c}2008 \\
(n=26350) \\
\%(I C 95 \%)\end{array}$ & $\begin{array}{c}2013 \\
(n=23815) \\
\%(I C 95 \%)\end{array}$ & $\begin{array}{c}2019 \\
(n=43554) \\
\%(I C 95 \%)\end{array}$ & $\begin{array}{c}\text { Valor } \\
\text { de p }\end{array}$ \\
\hline \multicolumn{5}{|c|}{ O seu domicílio está cadastrado (registrado) na unidade de Saúde da Família } \\
\hline Norte & $65(58,7-70,8)$ & $65,6(60,7-70,3)$ & $77,3(74,7-79,8)$ & $<0,001$ \\
\hline Nordeste & $76,7(74-79,2)$ & $81,5(78,9-83,8)$ & $86,5(84,8-88,0)$ & $<0,001$ \\
\hline Sudeste & $50,6(47,8-53,4)$ & $68,3(64,1-72,2)$ & $67,5(63,6-71,2)$ & $<0,001$ \\
\hline Sul & $64,8(61,3-68,1)$ & $75,7(71,2-79,8)$ & $80,4(77,3-83,2)$ & $<0,001$ \\
\hline Centro-oeste & $67,8(64,1-71,3)$ & $74,9(70,9-78,5)$ & $82,4(79,3-85,2)$ & $<0,001$ \\
\hline \multicolumn{5}{|c|}{ Procurou algum lugar, serviço ou profissional de saúde para atendimento relacionado à própria saúde } \\
\hline Norte & $21,5(18,4-24,9)$ & $17,0(14,2-20,3)$ & $23,1(20,9-25,5)$ & 0,004 \\
\hline Nordeste & $19,1(17,7-20,6)$ & $19,7(17,7-21,9)$ & $22,5(20,9-24,2)$ & $<0,001$ \\
\hline Sudeste & $23,8(22,3-25,3)$ & $27,8(24,9-30,9)$ & $28,7(26,3-31,1)$ & $<0,001$ \\
\hline Sul & $23,0(21,1-24,9)$ & $32,0(28,8-35,5)$ & $30,4(28,1-32,8)$ & $<0,001$ \\
\hline Centro-oeste & $23,5(20,5-26,9)$ & $24,6(21,4-28,2)$ & $26,6(23,8-29,5)$ & $<0,001$ \\
\hline
\end{tabular}

Foi atendido (a) nessa última vez que procurou atendimento de saúde, nas duas últimas semanas

$\begin{array}{lcccc}\text { Norte } & 56,7(13,6-91,6) & 35,0(7,3-78,8) & 82,2(70,3-90,0) & 0,032 \\ \text { Nordeste } & 48,3(26,5-70,8) & 38,4(20,4-60,4) & 78,1(68,9-85,1) & <0,001 \\ \text { Sudeste } & 56,9(35,8-75,8) & 47,3(23,2-72,7) & 87,5(79,0-92,8) & <0,001 \\ \text { Sul } & 47,6(18,1-78,9) & 46,2(14,8-81,0) & 91,1(84,6-95,0) & 0,028 \\ \text { Centro-oeste } & 36,5(8,8-77,3) & 51,3(22,4-79,4) & 86,0(75,9-92,3) & 0,005\end{array}$

Por que motivo não foi atendido(a) nessa última vez que procurou atendimento de saúde nas duas últimas semanas?

\begin{tabular}{|c|c|c|c|c|}
\hline \multicolumn{5}{|l|}{ Norte } \\
\hline Não conseguiu vaga nem pegar senha & - & $91,0(51,9-99,0)$ & $21,2(8,1-44,9)$ & 0,232 \\
\hline Não tinha médico atendendo & $65,7(10,7-96,8)$ & $9,0(1,0-48,1)$ & $32,4(13,0-60,6)$ & \\
\hline $\begin{array}{l}\text { Não tinha serviço ou profissional } \\
\text { especializado }\end{array}$ & $34,3(3,2-89,3)$ & - & $25,7(4,7-70,8)$ & \\
\hline Esperou muito e desistiu & - & - & $12,1(2,8-39,7)$ & \\
\hline $\begin{array}{l}\text { O serviço de saúde não estava } \\
\text { funcionando }\end{array}$ & - & - & $6,5(1,3-27,3)$ & \\
\hline $\begin{array}{l}\text { Os equipamentos do serviço de saúde } \\
\text { não estavam funcionando ou disponíveis } \\
\text { para uso }\end{array}$ & - & - & $2,1(0,3-14,6)$ & \\
\hline \multicolumn{5}{|l|}{ Nordeste } \\
\hline Não conseguiu vaga ou senha & $24,8(5,2-66,4)$ & $13,4(4,4-34,4)$ & $35,7(20,4-54,7)$ & 0,087 \\
\hline Não tinha médico atendendo & $58,3(24,6-85,7)$ & $40,5(15,7-71,5)$ & $42,6(24,5-63,0)$ & \\
\hline $\begin{array}{l}\text { Não havia serviço ou profissional de } \\
\text { saúde especializado para atender }\end{array}$ & - & - & $3,1(0,9-9,9)$ & \\
\hline Esperou muito e desistiu & $11,8(1,6-52,3)$ & $34(10,7-68,8)$ & $2,6(0,4-16,6)$ & \\
\hline $\begin{array}{l}\text { O serviço de saúde não estava } \\
\text { funcionando }\end{array}$ & - & $2,9(0,4-19,3)$ & $4,7(1,1-18,1)$ & \\
\hline
\end{tabular}

$\mathrm{de}^{15,16}$. Dessa forma, a realização de um inquérito específico para a saúde provavelmente favoreceu uma maior precisão das estimativas nas PNS. Porém, é imprescindível considerar que, apesar das referidas disparidades, esta pesquisa foi elaborada para permitir o monitoramento da maior parte dos indicadores elencados na PNAD $2008^{15}$. 
Tabela 3. Descrição temporal das variáveis relativas às morbidades, acesso e utilização de serviços de saúde na população idosa brasileira, por região geográfica, 2019.

\begin{tabular}{|c|c|c|c|c|}
\hline & $\begin{array}{c}2008 \\
(\mathrm{n}=26350) \\
\%(\mathrm{IC} 95 \%) \\
\end{array}$ & $\begin{array}{c}2013 \\
(\mathbf{n}=23815) \\
\%(\mathrm{IC} 95 \%) \\
\end{array}$ & $\begin{array}{c}2019 \\
(\mathrm{n}=43554) \\
\%(\mathrm{IC} 95 \%) \\
\end{array}$ & $\begin{array}{l}\text { Valor } \\
\text { de p }\end{array}$ \\
\hline $\begin{array}{l}\text { Os equipamentos do serviço de saúde } \\
\text { não estavam funcionando ou disponíveis } \\
\text { para uso }\end{array}$ & - & $9,2(1,3-44,5)$ & $8,8(1,9-33,3)$ & \\
\hline Outro motivo & $5,1(0,7-30,4)$ & - & $2,3(0,5-9,6)$ & \\
\hline \multicolumn{5}{|l|}{ Sudeste } \\
\hline Não conseguiu vaga ou senha & $15,8(3,8-47,2)$ & $7,6(1,1-38,4)$ & $8,0(2,2-25)$ & 0,400 \\
\hline Não tinha médico atendendo & $70,4(40,2-89,4)$ & $86,5(56,7-96,9)$ & $43,4(19,6-70,8)$ & \\
\hline $\begin{array}{l}\text { Não tinha serviço ou profissional } \\
\text { especializado }\end{array}$ & $7(0,9-37,4)$ & - & $20,6(6,5-49,1)$ & \\
\hline Esperou muito e desistiu & - & - & $0,7(0,1-5,0)$ & \\
\hline $\begin{array}{l}\text { O serviço ou equipamento não estava } \\
\text { funcionando }\end{array}$ & $6,8(0,9-36,8)$ & - & $9,8(1,4-46,1)$ & \\
\hline Outro & - & $5,8(0,8-32)$ & $17,4(5,9-41,7)$ & \\
\hline \multicolumn{5}{|l|}{ Sul } \\
\hline Não conseguiu vaga ou senha & $40,4(7,9-48,3)$ & $58,6(12,3-93,4)$ & $48(19,6-77,8)$ & ** \\
\hline $\begin{array}{l}\text { Não tinha serviço ou profissional } \\
\text { especializado }\end{array}$ & $59,6(15,7-92,1)$ & $41,4(6,6-87,7)$ & $39,2(14,1-71,6)$ & \\
\hline Esperou muito e desistiu & - & - & $12,8(1,7-55,3)$ & \\
\hline \multicolumn{5}{|l|}{ Centro-Oeste } \\
\hline Não conseguiu vaga ou senha & $17,5(2,2-67,2)$ & $67,2(24,2-92,9)$ & $19,2(5,7-48,2)$ & ** \\
\hline Não tinha médico atendendo & $82,5(32,8-97,8)$ & $32,8(7,1-75,8)$ & $22,0(7,8-48,4)$ & \\
\hline Esperou muito e desistiu & - & - & $58,9(29,2-83,2)$ & \\
\hline \multicolumn{5}{|l|}{ Hipertensão arterial } \\
\hline Norte & $50,2(44,3-56,0)$ & $45,9(39,2-52,6)$ & $47,4(43,8-51,1)$ & 0,310 \\
\hline Nordeste & $53,8(51,8-55,7)$ & $48,3(43,2-53,5)$ & $58,8(55,7-61,8)$ & $<0,001$ \\
\hline Sudeste & $60,1(58,4-61,9)$ & $60,5(55,5-65,4)$ & $59,7(56,1-63,3)$ & 0,195 \\
\hline Sul & $57,1(54,1-60,0)$ & $56,1(51,0-61,2)$ & $59,8(56,4-63,1)$ & 0,071 \\
\hline Centro-oeste & $55,3(52,1-58,5)$ & $54,6(48,1-61,0)$ & $62,3(57,3-66,9)$ & 0,042 \\
\hline \multicolumn{5}{|l|}{ Diabetes } \\
\hline Norte & $14,9(12,4-17,8)$ & $16,8(12,4-22,2)$ & $18,2(15,2-21,6)$ & 0,009 \\
\hline Nordeste & $13,9(12,7-15,2)$ & $17,5(14,0-21,6)$ & $20,1(17,9-22,6)$ & $<0,001$ \\
\hline Sudeste & $19,4(18,1-20,7)$ & $23,6(19,8-27,8)$ & $25,3(22,4-28,3)$ & $<0,001$ \\
\hline Sul & $16,2(14,3-18,4)$ & $19,8(16,2-23,9)$ & $20,9(18,2-23,9)$ & 0,003 \\
\hline Centro-oeste & $16,5(14,2-19)$ & $23,8(18,5-30,0)$ & $25,3(21,2-30,0)$ & $<0,001$ \\
\hline \multicolumn{5}{|l|}{ Multimorbidade } \\
\hline Norte & $48,6(43,9-53,2)$ & $44,4(37,5-51,5)$ & $53,0(48,8-57,1)$ & 0,004 \\
\hline Nordeste & $44,4(42,3-46,5)$ & $48,7(43,2-54,2)$ & $57,3(54,2-60,4)$ & $<0,001$ \\
\hline Sudeste & $50,7(48,9-52,5)$ & $61,8(56,7-66,6)$ & $63,8(60,5-67,1)$ & $<0,001$ \\
\hline Sul & $55,3(52,7-57,9)$ & $62,1(56,4-67,4)$ & $65,5(62,4-68,4)$ & $<0,001$ \\
\hline Centro-oeste & $54,7(51,6-57,8)$ & $51,6(44,8-58,4)$ & $60,7(56,0-65,3)$ & $<0,001$ \\
\hline
\end{tabular}

** categorias incapazes de comparações pela limitação do teste.

Fonte: Pesquisa Nacional por Amostra de Domicílios (2008). Pesquisa Nacional de Saúde 2013 e 2019.

so de transição demográfica, que se iniciou no Brasil na década de 1940 com a queda da mortalidade pelos avanços nas condições de vida, impulsionados pela Revolução Industrial e urbanização, resultando no aumento da expectativa de vida; e seguiu com a queda da fecundidade 
a partir da década de 1960. Assim, estes fatores provocaram mudanças na estrutura etária da população e maior proporção de pessoas idosas, de modo que a Organização das Nações Unidas (ONU) considera o período corrente de 1975 a 2025 como a Era do Envelhecimento ${ }^{18,19}$.

A despeito do aumento do nível de cobertura da APS em todas as regiões ao longo do mesmo período analisado, variando no Brasil de 64,32\% em 2008 para 70,58\% em 2013 e 74,76\% em 2019, segundo dados do Ministério da Saúde ${ }^{20}$, foi, contraditoriamente, verificado no presente estudo um decréscimo na procura pela APS pelos idosos brasileiros. Sincronicamente a esta tendência, cresceu a busca pelas unidades de Urgência e Emergência, o que pode ser explicado pela ênfase da implantação das Unidades de Pronto Atendimento (UPA) a partir de 2009. A utilização dos estabelecimentos de saúde depende, em parte, da percepção de necessidade pelos indivíduos e, considerando que as UPA representam unidades intermediárias entre a atenção primária e as emergências hospitalares, infere-se que muitos usuários passaram a procurar diretamente as UPA para assistência às necessidades de urgência, mesmo que muitas destas possam ser atendidas pela APS $^{21,22}$. Dessa forma ocorre o estabelecimento de uma atenção em saúde desvinculada da longitudinalidade do cuidado, promoção da saúde e prevenção de doenças e, por conseguinte, fragilização e redução da resolutividade da APS; além de uma sobrecarga e desvio de finalidade das UPAS, prejudicando o seu potencial de atuação assistencial.

Destaca-se que a APS não deve estar excluída deste processo de assistência no âmbito das UPA, mas sim articulada, de modo a garantir a longitudinalidade e coordenação do cuidado. Ademais, a rede de saúde deve estar atenta para que não se incentive o modelo curativista e hospitalocêntrico em detrimento das ações de promoção e prevenção em saúde, promovidas especialmente pela APS e fundamentais para o enfrentamento da maior carga de DCNT na contemporaneidade, aqui comprovada pelo aumento nas prevalências das morbidades diabetes mellitus, hipertensão arterial sistêmica e da ocorrência de multimorbidades $^{23}$.

Este perfil de saúde da população idosa atual, assim como ocorre nos dados da população total, tem se alterado em função das já citadas mudanças na estrutura etária, características da transição demográfica, associadas a fatores econômicos, sociais, culturais e ambientais, promovendo o que Omran ${ }^{24}$ conceitua como transição epidemiológica. Esta é definida como uma redução da morbimortalidade por doenças infecciosas e crescimento da importância das DCNT, sendo fundamental considerarmos a presença de várias morbidades simultaneamente (multimorbidades) na atenção a esta demanda ${ }^{25}$.

O aumento da prevalência das DCNT no estudo, além de representar uma tendência, pode estar relacionada à maior oportunidade de diagnóstico das mesmas, fazendo-se fundamental o fortalecimento da APS para atendimento eficaz de toda a demanda existente ${ }^{26}$.

Observando-se outros tipos de serviço de saúde, os de âmbito privado, dados da Agência Nacional de Saúde Suplementar (ANS) mostram que a taxa de cobertura por planos privados de Saúde apresentou um crescimento entre os anos de 2008 (21,87 \%) e 2013 (25,4\% ), enquanto em 2019 se observa uma leve redução $(24,2 \%)^{27,28}$. Infere-se que este cenário tenha contribuído para a observada redução, ao longo dos inquéritos, da busca pela APS pelas camadas mais favorecidas socioeconomicamente: pessoas da raça/cor branca e na região Sudeste.

$\mathrm{Na}$ análise das regiões, sabe-se que as desigualdades regionais são características presentes na realidade brasileira e refletem iniquidades na atenção à saúde. As regiões Norte e Nordeste costumam apresentar resultados mais desfavoráveis de indicadores de saúde, como pior avaliação do estado de saúde, maior restrição de atividades, e menor uso de serviços de saúde ${ }^{26}$. Este cenário se observa no resultado de menor procura pela APS na região Norte. No entanto, o mesmo achado no Sudeste contradiz a tendência de melhores indicadores. Esta situação do Sudeste pode ser explicada pela menor cobertura da APS entre as regiões brasileiras, calculada em $68,84 \%$ no mês de outubro de 2020 pelo Ministério da Saúde ${ }^{20}$, e com a maior cobertura de planos privados entre as pessoas idosas, 33,1\% em 2015, segundo dados da Agência Nacional de Saúde Suplementar ${ }^{30}$, quando comparado às demais regiões brasileiras.

A raça/cor constitui um importante eixo de desigualdade, sendo os indivíduos pretos, pardos e indígenas aqueles com indicadores mais desfavoráveis de escolaridade, trabalho, acesso a bens e serviços, incluindo os de saúde ${ }^{31}$. Os achados do presente estudo mostraram um resultado diferente: menor procura à APS pelas pessoas de raça/cor branca; o que se deve à metodologia utilizada, com recorte para este tipo de estabelecimento especificamente.

A APS constitui um serviço predominantemente público, sendo identificado que 99,2\% 
destes pertenciam ao SUS em $2017^{8}$. Portanto, temos que a população de raça/cor preta ou parda é mais dependente do SUS, enquanto que uma relevante parcela das pessoas de raça/cor branca possuem um plano de saúde privado, a qual correspondeu a $53,1 \%$ das pessoas idosas brancas, de acordo com Silva et al. ${ }^{32}$ no Estudo Saúde, Bem-Estar e Envelhecimento realizado na cidade de São Paulo/SP. Tal dado reflete a realidade das iniquidades em saúde determinadas pela raça, a qual está incluída no conceito de determinantes sociais de saúde como um fator estrutural da hierarquização social, interferindo nos desfechos de saúde, doença e morte ${ }^{33}$.

O considerável aumento no número de domicílios cadastrados na USF, favorece os achados deste estudo ao identificar que a população idosa investigada, quando abordada sobre a busca recente por serviços de saúde, procurou mais por atendimento em saúde ao longo do período e teve um vultoso êxito em consegui-lo no ano de 2019. Esta variável apresentou uma evolução inversamente proporcional à ausência de médico como justificativa nos casos que não conseguiram atendimento, com o surgimento de números mais desfavoráveis de 2008 a 2013, porém com um relevante quadro positivo em 2019. Portanto, além da expansão da APS em si, infere-se que a maior disponibilidade de profissionais médicos contribui para o resultado dos usuários lograrem atendimento, podendo estas conquistas serem explicadas pela criação e implantação do Programa mais médicos, o qual tem por objetivo diminuir as desigualdades regionais decorrentes da carência desses profissionais e fortalecer a atenção primária como eixo coordenador da atenção à saúde ${ }^{34}$.

Ainda assim, a falta de médicos representa a maior barreira para o atendimento na população idosa, havendo insuficiência de profissionais para a atenção a toda demanda existente. Esta realidade agrava a dificuldade de acesso ao serviço, visto que foi constatado que a dificuldade em conseguir vaga ou senha, possivelmente devido ao reduzido quantitativo de profissionais aptos para suprir a demanda existente, foi o segundo motivo mais frequente de não atendimento nos anos de 2008, 2013 e 2019, com considerável aumento na região Nordeste, em detrimento do verificado nas demais regiões. Ressalta-se que o direcionamento de atendimentos pela distribuição de senhas pode gerar um impacto negativo na concretização do acesso e utilização de serviços de saúde, pois, conforme ressaltam Nunes et al. ${ }^{22}$, esse modelo estimula a cultura de formação de extensas filas, anteriormente ao início do horário de atendimento nas UBS, no intuito de garantir a assistência em saúde e concretização da utilização do serviço de saúde.

Considerando que se trata de um estudo transversal em painéis, elencamos como limitações: suscetibilidade ao viés de sobrevivência; além de, ambas as pesquisas, o Suplemento Saúde da PNAD 2008 e PNS desconsiderarem a população residente em instituições coletivas, em embaixadas, consulados e, também, as pessoas institucionalizadas residentes em domicílios coletivos de estabelecimentos institucionais, tais como: os militares em caserna ou dependências de instalações militares; os presos em penitenciárias; os internos em escolas, orfanatos, asilos, hospitais, os religiosos em conventos, mosteiros. Porém, este estudo caracteriza-se por ser de abrangência nacional, o que permite identificar as condições e tendências de acesso e utilização aos serviços de saúde na APS pela população idosa brasileira.

\section{Considerações finais}

A transição epidemiológica vigente no Brasil pôde ser ratificada com a identificação de uma tendência de crescimento nas prevalências das morbidades hipertensão arterial sistêmica, diabetes mellitus e da ocorrência de multimorbidade entre os dados da PNAD 2008 e PNS 2019, reafirmando a necessidade de acompanhamento e mobilização para o alcance das metas pactuadas no Plano de enfrentamento das doenças crônicas não transmissíveis 2011-2022.

A APS é considerada como um dos mecanismos essenciais para o enfrentamento da DCNT, de maneira que a constatação de ampliação no cadastro das USF, associada a maior procura e atendimento neste nível de atenção reforça a relevância da consolidação da APS para que seja possível qualificação na atenção à saúde aos idosos brasileiros, em prol de uma população idosa mais saudável.

Em consonância com esta considerável expansão da APS no presente estudo, ao identificar o aumento no acesso e utilização dos serviços de saúde da APS em todas as regiões brasileiras, é possível elencar o relevante papel das diversas políticas que fomentam o cuidado em saúde aos idosos, favorecendo impactos positivos na garantia ao acesso e utilização dos serviços de saúde, assim como no acompanhamento desses idosos em quaisquer regiões do país, conforme identificados nesta pesquisa. 


\section{Colaboradores}

VAC Cesário e TCO Mendes atuaram nas etapas de planejamento, coleta de dados, interpretação dos resultados e elaboração do texto. MM Santos participou do planejamento do estudo, análise, interpretação e escrita dos resultados. KC Lima contribui com o planejamento teórico-metodológico. Todos os autores realizaram revisão crítica e aprovação da versão final do manuscrito.

\section{Referências}

1. World Health Organization (WHO). Global status report on noncommunicable diseases 2014 [Internet]. Geneva: WHO; 2014 [cited 2018 May 23]. Available from: http://apps.who.int/iris/bitstream/ handle/10665/148114/9789241564854_eng.pdf;jsessionid=270C4B4F9FFB5EFB42BE5721D01FF290?sequence $=1$

2. Nunes BP, Batista SRR, Andrade FB, Souza Junior PRB, Lima-Costa MF, Facchini LA. Multimorbidity: The Brazilian Longitudinal Study of Aging (ELSI-Brazil). Rev Saude Publica 2018; 52(Supl. 2):10s.

3. Harrison C, Britt H, Miller G, Henderson J. Examining different measures of multimorbidity, using a large prospective cross-sectional study in Australian general practice. BMJ Open 2014; 4(7):e004694.

4. Souza MFM, Malta DC, França EB, Barreto ML. Changes in health and disease in Brazil and its States in the 30 years since the Unified Healthcare System (SUS) was created. Cien Saude Colet 2018; 23(6):17371750.

5. Veras R. O modelo assistencial contemporâneo e inovador para os idosos. Rev Bras Geriatr Gerontol 2020; 23(1):e200061.

6. Schenker M, Costa DH. Advances and challenges of health care of the elderly population with chronic diseases in Primary Health Care. Cien Saude Colet 2019; 24(4):1369-1380.

7. Brasil. Portaria n⿳2 2436 de 21 de setembro de 2017. Aprova a Política Nacional de Atenção Básica, estabelecendo a revisão de diretrizes para a organização da Atenção Básica, no âmbito do Sistema Único de Saúde (SUS). Diário Oficial da União 2017; 21 set.

8. Viacava F, Oliveira RAD, Carvalho CC, Laguardia J, Bellido JG. SUS: oferta, acesso e utilização de serviços de saúde nos últimos 30 anos. Cien Saude Colet 2018; 23(6):1751-1762.

9. Bonello AADLM, Corrêa CRS. Acesso aos serviços básicos de saúde e fatores associados: Estudo de base populacional. Cien Saude Colet 2014; 19(11):4397-4406.

10. Cruz PKR, Vieira MA, Carneiro JÁ, Costa FM, Caldeira AP. Dificuldades do acesso aos serviços de saúde entre idosos não institucionalizados: prevalência e fatores associados. Rev Bras Geriatr Gerontol 2020; 23(6):1-13.

11. Bibiano AMB, Moreira RDS, Tenório MMGDO, Silva VDL. Factors associated with the use of the health services by elderly men: A systematic review of the literature. Cien Saude Colet 2019; 24(6):2263-2278.

12. Paula A, Coelho S, Pereira B, Iii N, Manjourany S, Duro S. Determinantes socioeconômicos do acesso a serviços de saúde em idosos: revisão sistemática. Rev Saude Publica 2017; 51:1-15.

13. Pedraza DF, Nobre AMD, de Albuquerque FJB, de Menezes TN. Acessibilidade às Unidades Básicas de Saúde da Família na perspectiva de idosos. Cien Saude Colet 2018;23(3):923-933.

14. Viacava F, Bellido J G. Condições de saúde, acesso a serviços e fontes de pagamento, segundo inquéritos domiciliares. Cien Saude Colet 2016; 21(2):351-370. 
15. Stopa SR, Szwarcwald CL, Oliveira MM, Gouvea ECDP, Vieira MLFP, Freitas MPS, Sardinha LMV, Macário EM. Pesquisa Nacional de Saúde 2019: histórico, métodos e perspectivas. Epidemiol Serv Saude 2020; 29(5):e2020315.

16. Silva VSTM, Pinto LF. Inquéritos domiciliares nacionais de base populacional em saúde: uma revisão narrativa Cien Saude Colet [Periódico na internet]. 2020 set [acessado 2020 Jan 27]. Disponível em: http:// www.cienciaesaudecoletiva.com.br/artigos/inqueritos-domiciliares-nacionais-de-base-populacional -em-saude-uma-revisao-narrativa/17780

17. Brasil. Instituto Brasileiro de Geografia e Estatística (IBGE). Projeção da População [Internet], 2021 [acessado 2021 jan 4]. Disponível em: https://sidra.ibge. gov.br/tabela/7358

18. Brasil. Instituto Brasileiro de Geografia e Estatística (IBGE). Mudança Demográfica no Brasil no Início do Século XXI: Subsidios para as projeções da população do Brasil e das Unidades da Federação. [Internet]. Rio de Janeiro: IBGE; 2015 [acessado em 2021 jan 04]. Disponível em: https://biblioteca.ibge.gov.br/visualizacao/livros/liv93322.pdf

19. Siqueira RL, Botelho MIV, Coelho FMG. A velhice: algumas considerações teóricas e conceituais. Cien Saude Colet 2002; 7(4):899-906.

20. Brasil. Ministério da Saúde (MS). Secretaria de Atenção Primária à Saúde - SAPS. Informação e Gestão da Atenção Básica - e-Gestor AB. [acesso 2021 jan 09] 2021. Disponível em: https://egestorab.saude.gov.br/ paginas/acessoPublico/relatorios/relHistoricoCoberturaAB.xhtml

21. O’Dwyer G, Konder MT, Reciputti LP, Lopes, MGM, Agostinho DF, Alves GF. O processo de implantação das unidades de pronto atendimento no Brasil. Rev Saude Publica 2017; 51:125.

22. Nunes BP, Flores TR, Garcia LP, Chiavegatto Filho ADP, Thumé E, Facchini LA. Tendência temporal da falta de acesso aos serviços de saúde no Brasil, 19982013. Epidemiol Serv Saude 2016; 25(4):777-787.

23. Uzuelli FHP, Costa ACD, Guedes B, Sabiá CF, Batista SRR. Reforma da Atenção Hospitalar para modelo de saúde baseada em valor e especialidades multifocais. Cien Saude Colet 2019; 24(6):2147-2154.

24. Omran AR. The epidemiologic transition: a theory of the epidemiology of population change. The Milbank Memorial Fund Quarterly 1971; 4(4):509-538.

25. Melo LA, Lima KC. Prevalência e fatores associados a multimorbidades em idosos brasileiros. Cien Saude Colet 2020;25(10):3869-3877.

26. Viacava F, Porto SM, Carvalho CC, Bellido JG. Desigualdades regionais e sociais em saúde segundo inquéritos domiciliares (Brasil, 1998-2013). Cien Saude Colet 2019; 24(7):2745-2760.
27. Brasil. Ministério da Saúde (MS). Rede Interagencial de Informações para a Saúde. Indicadores e Dados Básicos [Internet]. Brasil: DATASUS;2021 [acessado 2021 jan 9]. Disponível em: http://tabnet.datasus.gov.br/ cgi/tabcgi.exe?idb2012/f16.def.

28. Agência Nacional de Saúde Suplementar (ANS). Dados do Setor. [Internet]. 2021. [acessado 2021 jan 09]; [cerca de 1p.] Disponível em: https://www.ans.gov.br/ perfil-do-setor/dados-gerais

29. Brasil. Ministério da Saúde (MS). Secretaria de Atenção Primária à Saúde - SAPS. Informação e Gestão da Atenção Básica - e-Gestor AB. [acesso 2021 jan 9]. Disponível em: https://egestorab.saude.gov.br/paginas/ acessoPublico/relatorios/relHistoricoCoberturaAB. xhtml/

30. Agência Nacional de Saúde Suplementar (ANS). Idosos na Saúde Suplementar: uma urgência para a saúde da sociedade e sustentabilidade do setor. Projeto Idoso Bem Cuidado. Rio de Janeiro: ANS; 2016. [acessado 2021 jan 09]; [cerca de 134]. Disponível em: https:// www.ans.gov.br/images/stories/Materiais_para_pesquisa/Materiais_por_assunto/web_final_livro_idosos.pdf

31. Paixão M, Rossetto I, Montovanele F, Carvano LM, editores. Relatório anual das desigualdades raciais no Brasil: Constituição cidadã, seguridade social e seus efeitos sobre as assimetrias de cor ou raça. Rio de Janeiro: Garamond; 2011.

32. Silva A, Rosa TEC, Batista LE, Kalckmann E, Louvison MCP, Teixeira DSC, Lebrão ML. Iniquidades raciais e envelhecimento: análise da coorte 2010 do Estudo Saúde, Bem-Estar e Envelhecimento (SABE). Rev Bras Epidemiol 2019;21(Supl. 2): e180004.

33. Werneck J. Racismo institucional e saúde da população negra. Saude Soc 2016; 25(3):535-549.

34. Brasil. Lei $n^{\circ} 12.871$, de 22 de outubro de 2013. Institui o Programa Mais Médicos. Diário Oficial da União 2013; 23 out.

Artigo apresentado em 02/02/2021

Aprovado em 07/05/2021

Versão final apresentada em 09/05/2021

Editores-chefes: Maria Cecília de Souza Minayo, Romeu Gomes, Antônio Augusto Moura da Silva 